\title{
The Validity of a Parent-based Assessment of Cognitive Abilities in Three-year Olds*
}

\author{
BONAMY OLIVER ${ }^{a}$, PHILIP S. DALE ${ }^{b}$, KIMBERLY J. SAUDINO $^{c}$ \\ STEPHEN A. PETRILL ${ }^{c}$, ALISON PIKE $^{d}$ and ROBERT PLOMIN ${ }^{a, \dagger}$ \\ ${ }^{a}$ Social, Genetic and Developmental Psychiatry Research Centre; ${ }^{b}$ Communication Sciences and \\ Disorders; ${ }^{\mathrm{c}}$ Department of Psychology; ${ }^{\mathrm{d}}$ Psychology Group
}

\section{(Received 12 October 2001)}

\begin{abstract}
The purpose of the present study was to validate a parent-based assessment of cognitive abilities of three-yearold children against a standard tester-administered measure. The cognitive abilities of 85 children $(50$ boys and 35 girls, members of 43 twin pairs) were assessed using a measure of non-verbal abilities called the Parent Report of Children's Abilities for three-year-olds (PARCA3), a vocabulary checklist, and the McCarthy Scales of Children's Abilities (McCarthy, 1972). Correlations indicated that both components of the PARCA3 (parent-report and parent-administered) were significantly associated with the McCarthy scales, as was the vocabulary measure. Most importantly, the PARCA3 and vocabulary measure in combination significantly and substantially predict the McCarthy General Cognitive Index (Total $R=0.63$ ). We conclude that the PARCA3, with the vocabulary measure, provides a valid, inexpensive, reliable measure of cognitive ability for very young children.
\end{abstract}

Key words: Parents; Test validity; Cognitive ability; Cognitive assessment; Developmental measures; Cognitive development

The present study describes the development of a parent-based assessment of the verbal and non-verbal cognitive abilities of three-year-old children, and the validation of this measure. Although traditional tester-based measures of young children are broadly accepted, it has been argued that tester-based assessment is limited in what it can tell us about the typicality of the child's performance (Bornstein and Haynes, 1998). Our hypothesis, based on the literature and our previous work in this area, is that parent-based assessment can validly measure cognitive abilities in young children. Moreover, there are some distinct advantages associated with this form of testing.

Tester-based assessment requires that children perform their best for a stranger. Indeed, most testers will have heard a parent lamenting "his or her youngster's not saying (or doing) something with a stranger that the same child says (or does) often when with the parent alone" (Bornstein and Haynes, 1998, p. 655). This is often frustrating for a parent, but more importantly may reflect an inaccurate indication of a child's developmental level. Nonetheless, many tester-administered measures are now widely used in research and in clinics

\footnotetext{
* This research was supported by the UK Medical Research Council.

$\dagger$ Corresponding author. Present address: Social, Genetic and Developmental Psychiatry Research Centre, Institute of Psychiatry, 111 Denmark Hill, London SE5 8AF, UK. A copy of the PARCA3 can also be obtained at cost from Robert Plomin.
}

ISSN 0300-4430 print; ISSN 1476-8275 online (C) 2002 Taylor \& Francis Ltd DOI: $10.1080 / 0300443022000004648$ 
(e.g., the Stanford-Binet Intelligence Scale (Thorndike, Hagen and Sattler, 1986), and the Wechsler Preschool and Primary Scales of Intelligence (Wechsler, 1989)).

In contrast, although parent-based measures are used in many behavioural domains such as behavioural problems, temperament and family functioning, there are few examples of the use of parent-based measures for assessing cognitive ability in pre-school aged children. The assumption is that parent-based measures are prone to problems. In particular, parents might misinterpret the child's behaviour or the question and they might not have a sufficient frame of reference to accurately judge their child's performance (Fenson et al., 1994). However, the benefits of using parents' assessments include the fact that parents observe their children across time and in many situations and are of course familiar to the children, unlike a tester who is a stranger (Fenson et al., 1994).

Although there is some evidence that parents are able to report accurately about their children's abilities, thus far these parent-based measures assess the child's general development rather than specific aspects of cognitive ability. For example, these instruments assess a wider range of behaviours such as motor, social and language skills as well as cognitive abilities (e.g., Minnesota Child Development Inventory; Ireton and Thwing, 1974), and more recently Infant Monitoring Questionnaires (Bricker and Squires, 1989). In addition, previous measures have been based on parental reports of their children's abilities, rather than asking parents to actually administer developmental tasks.

The development of a parent-based assessment of children's language skills, the MacArthur Communication Development Inventory (Fenson, Pethick and Cox, 1994), clearly demonstrated that parents could provide reliable and accurate data about aspects of their child's language development. This measure has since been shortened, anglicised and used successfully in a U.K. population-based sample of 2-year-old twins participating in the Twins' Early Development Study (TEDS; Dale et al., 1998).

The impetus for the design of this shortened instrument was the need for information on the very large number of children participating in TEDS. TEDS is a longitudinal study that has been running since 1995 and involves over 15,000 pairs of twins born throughout England and Wales and their families. One of the principal aims of the study is to examine the cognitive and language abilities of these children in order to distinguish the genetic and environmental origins of verbal and non-verbal delay and behavioural problems. Given that it was not feasible to consider tester-administered measures of the abilities of all of these children, an alternative to these testing methods was sought.

The success of the parent report of language prompted an interest in the development of a parent-based measure of cognitive abilities for two year olds, the Parent Report of Children's Abilities (PARCA; Saudino et al., 1998). This measure of the non-verbal skills of two-year-olds, which included a parent questionnaire in addition to parentadministered tasks, indicated that parents are valuable sources of information about their child's non-verbal development, as well as their language development, certainly at this young age. A total PARCA score, derived from both the parent-report and the parentadministered components was found to predict performance on the Mental Development Index (MDI) of the Bayley Scales of Infant Development-II (BSID-II; Bayley, 1993) $(r=0.51, p<0.001)$.

Following on from these successes, then, similar measures of both verbal and nonverbal cognitive abilities designed for older children were developed. The aim of the present study was to examine the validity of these parent-based measures of three-year old children. Using comparisons with a well-established tester-based assessment, we investigated the criterion validity of parent-based measures of non-verbal and verbal ability. Our expectation was that the parent-based measures would discriminate between verbal and non-verbal abilities as assessed by the tester-based measures. 


\section{METHOD}

\section{Sample}

All children were twins participating in the Twins Early Development Study (TEDS). TEDS is a longitudinal study of twins born in 1994, 1995 and 1996 in England and Wales. Children in the present study were selected from the 1041 twins born in the last five months of 1995 for whom parent-based assessments at three-years were complete and returned to us within three months of the twins' birthdays. In addition, families were selected due to geographical convenience, that is locations where other TEDS testing was taking place throughout England and Wales. 96 children (48 twin pairs) were visited. Of these children, seven were excluded because of one of the following reasons: unwell at the time of the tester visit, refused to co-operate with the tester, missing McCarthy sub-tests, or English was not the first language spoken at home. Thus the final sample included 50 boys and 35 girls between the ages of 36 and 39 months at the time of in-home testing. The sample was predominantly white (98\% compared to the $92 \%$ figure for mothers with children born in 1994, according to the Office for National Statistics), and 36\% had completed A-level examinations (compared to $32 \%$ as reported by the Office for National Statistics).

\section{Measures}

\section{The McCarthy Scales of Children's Abilities}

The McCarthy Scales of Children's Abilities was used as the standard tester-administered assessment of cognitive ability. The McCarthy includes 5 sub-scales, Memory, Perceptual Performance, Verbal, Quantitative, and General Cognitive Index (GCI). In the present study, raw scores were used rather than age-adjusted index scores due to the narrow age-band of the children and to capitalise on the full variability of the children's scores. Previous research has demonstrated good reliability and validity for this measure (McCarthy, 1972).

\section{Parent Report of Children's Abilities (PARCA3)}

The successful design of a parental assessment of two-year old children's non-verbal abilities, the PARCA (Saudino et al., 1998), prompted the design of a similar measure for three-year olds (PARCA3). As with the two-year old version, the PARCA3 consists of a parent-report component and a parent-administered component.

The parent-report component consists of 22 questions that assess aspects of cognitive development such as spatial ability, planning and organising, reasoning, memory, quantitative skills, pretend play, and number. Since the PARCA3 aims to separate non-verbal ability from verbal skills, a particular effort was made to select items without any direct or indirect focus on language. 15 of the easiest questions from the original PARCA (those questions for which over $80 \%$ of two year-olds were able to do the activity) were not included in the PARCA3. Thus 11 questions were retained for the PARCA3. Other questions were adapted from existing standardised measures such as the Minnesota Child Development Inventory (Ireton and Thwing, 1974), and the Ages and Stages Questionnaires (Bricker, Squires and Mounts, 1995), as well as other novel items designed specifically for PARCA3.

For each question parents are asked to indicate whether or not they have seen their child perform the activity. For example, "Does your child draw simple pictures that other people can recognise, such as a person, house or car?" Parents are asked to answer "YES" or "NO" where possible, although a "DON'T KNOW" response was included. "YES" responses are 

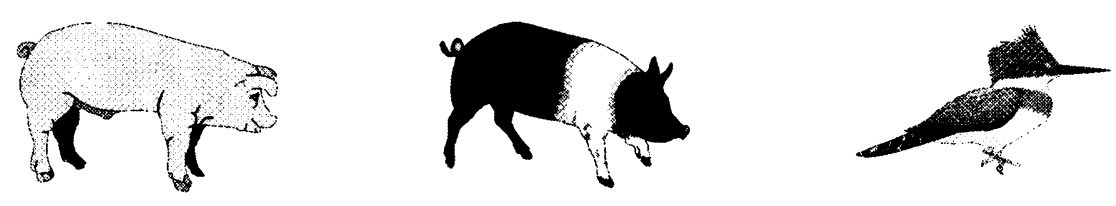

FIGURE 1 Sample item from the PARCA3 sub-scale "Find the Pair", in which the parent asks the child which two of three pictures "belong" together.

scored as 1 and "NO" and "DON'T KNOW" are scored as 0 . The total score for the parentreport component is derived by summing the items, so that the maximum score is 22 . Internal consistency as estimated by Cronbach's alpha was 0.67 , indicating acceptable reliability considering the breadth of the items.

As with the parent-report component of the PARCA3, the parent-administered component consists of some items from the original PARCA (those items for which fewer than $80 \%$ of two-year olds were successful), some items adapted from existing non-verbal items from various standard measures of cognitive ability (e.g. McCarthy Scales of Children's Abilities (McCarthy, 1972), BSID-II (Bayley, 1993)), and some items designed specifically for the PARCA3. Items were also selected to be enjoyable for children of this age and straightforward for parents to administer from written instructions.

The parent-administered component consists of three types of task, "Find the Pair", "Drawing" and "Matching" tasks. Within each set of tasks, there is a progression of difficulty so that the first item is relatively easy and later items much more difficult. The sub-scale "Find the Pair" asks the child to tell the parent which two of three pictures "belong" together (for an example see Fig. 1). The 14 items were designed to tap number, shape, conceptual grouping, and orientation skills. The "Drawing" sub-scale first asks the child to copy the parent's example of simple drawn designs (for example, a circle; a total of three designs) and then moves on to ask the child to copy an existing design (a further three designs). Finally, the "Matching" sub-scale asks the child to find a target shape among four alternatives (for an example, see Fig. 2). These 14 items assess various aspects of cognitive ability including conceptual grouping, number, size, and orientation.

Parents are asked to administer the items and record the child's responses. "Find the Pair" items and "Matching" sub-scales are scored 1 for a correct response and 0 for an incorrect

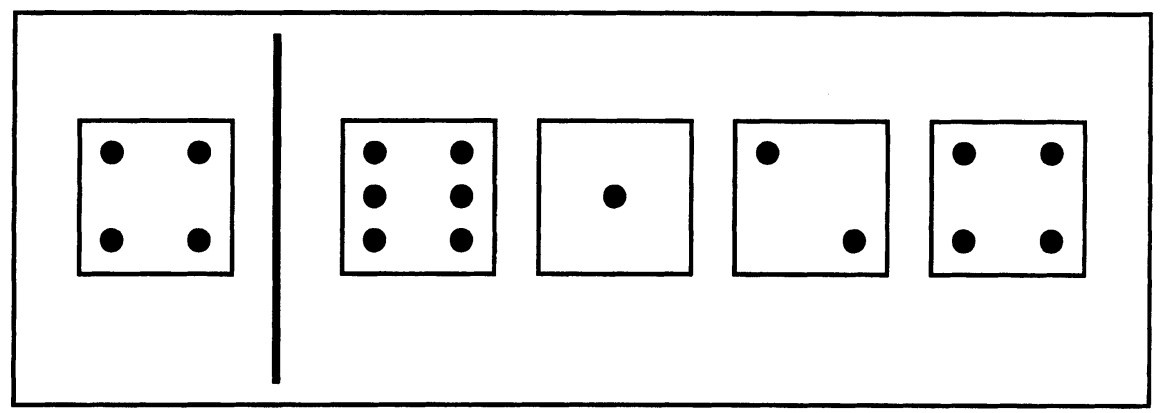

FIGURE 2 Sample item from the PARCA3 sub-scale "Matching", in which the child is asked to find a target shape among four alternatives. 
response. "Drawing" items are scored according to decision rules similar to those used when scoring standardised drawing tasks yielding scores between 0 and 2 . A total score for the parent-administered component is derived by summing across the three sub-scales following standardisation of each sub-scale to a mean of 0 and a standard deviation of 1 . Internal consistency of the total score as estimated by Cronbach's alpha was excellent, 0.83 .

Parent-report and parent-administered components are moderately correlated $(r=0.29$, $p<0.01)$. A total PARCA3 score was generated for each child by standardising total parentreport scores and parent-administered scores and summing them. Internal consistency for the total score was also 0.83 .

\section{Parent Report of Vocabulary}

The parent report of their children's language development was based on the success of the UK short form of MacArthur Communicative Development Inventories: Words and Sentences (MCDI:UKSF) used at 2 years (Saudino et al., 1998; Dale et al., 1998). The present study utilises the vocabulary component of an upward extension of the MCDI, the CDI-III, developed by Philip Dale, with similar form and design principles (Dale, Reznick and Thai, 1998). The CDI-III includes a 100 -item vocabulary checklist, which was taken as the clearest indicator of verbal ability (Sattler, 1988). The selection of words for inclusion on this list was based on the need for an appropriate overall level of difficulty, variability in word difficulty to ensure sensitivity to individual differences, and minimal overlap with the list used at 2 years. The 100 words included 3 words from the two-year MCDI:UKSF, 42 other words from the original, full MCDI, and 55 new words drawn from a variety of tests and the child language literature. Parents were asked to tick off those words they had heard their child say. The total number of items ticked comprised the vocabulary score for each child. Items included all parts of speech (e.g. dinosaur, then, today, angry, because). The internal consistency was excellent (alpha $=0.98)$.

\section{Feasibility}

A sample of 20 families with children around the age of three years (age range 32-40 months) was sought for a small feasibility test. Given that the feasibility testing involved children with at least average ability, the age range was deliberately broad to simulate an ability range. Families were volunteers recruited through TEDS staff and through parents who had previously helped with the pilot of the two-year-old PARCA (Saudino et al., 1998). Parents were sent a preliminary version of the PARCA3 for their child through the post, and asked to return the booklet, with comments, when completed. On the basis of this feedback, and the data from these families, the preliminary PARCA3 was revised to its final form, as used in the present study. The small changes made to the preliminary version consisted of revisions to instructions to aid clarity, as well as the deletion of items that were too difficult for all children, thus demonstrating no variability.

\section{Procedure}

Families were posted the PARCA3 and the vocabulary measure two weeks before the twins' third birthdays, and were asked to complete the booklets as near to the twins' birthdays as possible. When the booklets were complete, parents returned them to us for scoring. The twins were visited at home within three months of their birthdays and tested separately using the McCarthy Scales of Children's Abilities. 


\section{RESULTS}

\section{Preliminary Analyses}

The means and standard deviations for each of the measures used are presented in Table I. In order to utilise the power of the entire sample, the twin data were pooled for subsequent analyses, and these results are presented in the body of the text.

As expected, age was not correlated with any of the McCarthy scales, however a moderate correlation with age emerged for the Parent Report component of the PARCA3 $(r=0.28$, $p<0.01)$. There were no significant differences between scores for boys and girls; thus the entire sample was used for the remaining analyses.

\section{Correlation Analyses}

Table II contains the correlations between the PARCA3 scales, vocabulary and the each of the McCarthy sub-scales (verbal, perceptual performance, quantitative, memory). Both the parent-report and parent-administered components of PARCA3 were moderately to substantially associated with performance on all of these McCarthy scales $(r=0.22-0.43)$. Reassuringly, the lowest correlations emerged for the verbal scale. In general the parentreport component was more highly associated with the McCarthy scales than the parent-administered component. As expected, PARCA3 total scores were more highly correlated with McCarthy scales $(r=0.33-0.49)$, that is, in every case the correlation for the total PARCA3 score exceeded that of either of the individual components considered separately. In addition, a McCarthy non-verbal composite was calculated

TABLE I Means and (Standard Deviations) for McCarthy Scales and Parent Measures.

\begin{tabular}{lccccc}
\hline & $\begin{array}{c}\text { First-born } \\
(n=43)\end{array}$ & $\begin{array}{l}\text { Second-born } \\
(n=42)\end{array}$ & $\begin{array}{l}\text { Boys } \\
(n=50)\end{array}$ & $\begin{array}{l}\text { Girls } \\
(n=35)\end{array}$ & $\begin{array}{c}\text { Total } \\
(n=85)\end{array}$ \\
\hline McCarthy & & & & & \\
Verbal & 20.98 & 22.31 & 20.88 & 22.71 & 21.64 \\
Perceptual performance & $(10.96)$ & $(10.93)$ & $(11.20)$ & $(10.53)$ & $(10.90)$ \\
& 16.53 & 15.43 & 15.56 & 16.60 & 15.99 \\
Quantitative & $(6.53)$ & $(6.22)$ & $(5.85)$ & $(7.09)$ & $(6.37)$ \\
& 8.30 & 8.98 & 8.70 & 8.54 & 8.64 \\
Memory & $(5.19)$ & $(4.85)$ & $(5.17)$ & $(4.84)$ & $(5.01)$ \\
& 8.19 & 8.95 & 8.54 & 8.60 & 8.56 \\
GCI & $(5.54)$ & $(6.10)$ & $(5.84)$ & $(5.84)$ & $(5.80)$ \\
& 45.81 & 46.71 & 45.14 & 47.86 & 46.26 \\
NVMc* & $(20.04)$ & $(17.86)$ & $(18.82)$ & $(19.14)$ & $(18.89)$ \\
& 24.84 & 24.40 & 24.26 & 25.14 & 24.62 \\
PARCA3 & $(10.37)$ & $(9.04)$ & $(9.20)$ & $(10.45)$ & $(9.68)$ \\
Parent-report & & & & & \\
& 13.93 & 13.50 & 13.44 & 14.11 & 13.72 \\
Parent-administered & $(3.06)$ & $(3.01)$ & $(3.16)$ & $(2.81)$ & $(3.02)$ \\
& 0.08 & -0.08 & -0.32 & 0.45 & 0.00 \\
PARCA3 total score & $(2.45)$ & $(2.12)$ & $(1.84)$ & $(2.77)$ & $(2.28)$ \\
& 0.10 & -0.10 & -0.19 & 0.28 & 0.00 \\
Vocab & $(1.70)$ & $(1.53)$ & $(1.54)$ & $(1.69)$ & $(1.61)$ \\
& 58.67 & 55.79 & 55.38 & 59.91 & 57.25 \\
& $(25.88)$ & $(25.37)$ & $(26.80)$ & $(23.69)$ & $(25.52)$ \\
\hline
\end{tabular}

*Nonverbal ability score (NVMc) is a composite of Perceptual Performance, and Quantitative sub-scales. 
TABLE II Correlations Between Parent Measures and the McCarthy Scales.

\begin{tabular}{lcccc}
\hline & \multicolumn{4}{c}{ Parent measures } \\
\cline { 2 - 5 } & Parent-report & Parent-administered & PARCA3 total score & Vocab \\
\hline Verbal & $0.31^{* *}$ & $0.22^{*}$ & $0.33^{* *}$ & $0.57^{* * *}$ \\
Perceptual performance & $0.41^{* * *}$ & $0.28^{* *}$ & $0.43^{* * *}$ & $0.44^{* * *}$ \\
Quantitative & $0.43^{* * *}$ & $0.36^{* *}$ & $0.49^{* * *}$ & $0.52^{* * *}$ \\
Memory & $0.35^{* *}$ & $0.28^{*}$ & $0.39^{* * *}$ & $0.52^{* * *}$ \\
NVMc & $0.49^{* * *}$ & $0.38^{* * *}$ & $0.54^{* * *}$ & $0.56^{* * *}$ \\
GCI & $0.43^{* * *}$ & $0.32^{* *}$ & $0.46^{* * *}$ & $0.62^{* * *}$ \\
\hline
\end{tabular}

Note: ${ }^{*} p<0.05 ;{ }^{* *} p<0.01 ;{ }^{* * *} p<0.001$.

(NVMc) from the quantitative and perceptual performance scales. This was used as well as the GCI scale, which includes a verbal component. Although all correlations were substantial, excluding the verbal component of the McCarthy increased the associations. The highest correlation emerged between the NVMc and the PARCA3 total score $(r=0.54)$.

Correlations between all the McCarthy scales and vocabulary are also included in Table II. As expected, the highest correlation here was with the verbal sub-scale $(r=0.57)$, and GCI $(r=0.62)$. Surprisingly, however, the correlations obtained with the non-verbal scales were also quite substantial. In fact, vocabulary was more highly correlated than were the PARCA3 scales in every case. This is considered in the discussion. (All correlation analyses were also conducted separately for each twin, see Appendix).

\section{Regression Analyses}

Multiple regression analyses predicting the McCarthy scales from the PARCA3 total score and vocabulary were conducted and are presented in Table III. All of these regressions were highly significant. The multiple correlations were substantial, and ranged from 0.49 (perceptual performance) to 0.63 (GCI). Vocabulary was an independent predictor for every scale, while the PARCA3 total score was only an independent predictor for the non-verbal sub-scales, perceptual performance, quantitative and NVMc. Thus, in the case of the nonverbal scales the combination of the PARCA3 total score and vocabulary provided better prediction than either of these components separately. However, the PARCA3 total score did not aid in the prediction of the remaining three McCarthy scales, which all included a verbal component. (All regression analyses were also conducted separately for each twin and yielded similar results, see Appendix).

\section{DISCUSSION}

The aim of the study was to investigate the validity of a parent-based measure of cognitive abilities (PARCA3 and vocabulary). Firstly, we examined the criterion validity of the parentbased measures of verbal and non-verbal ability against a standard tester-administered measure (McCarthy). Secondly, the discriminant validity of the parent-based non-verbal and vocabulary measures was considered. That is, the differential contribution of the non-verbal and verbal measures to the prediction of the McCarthy scales was assessed. 


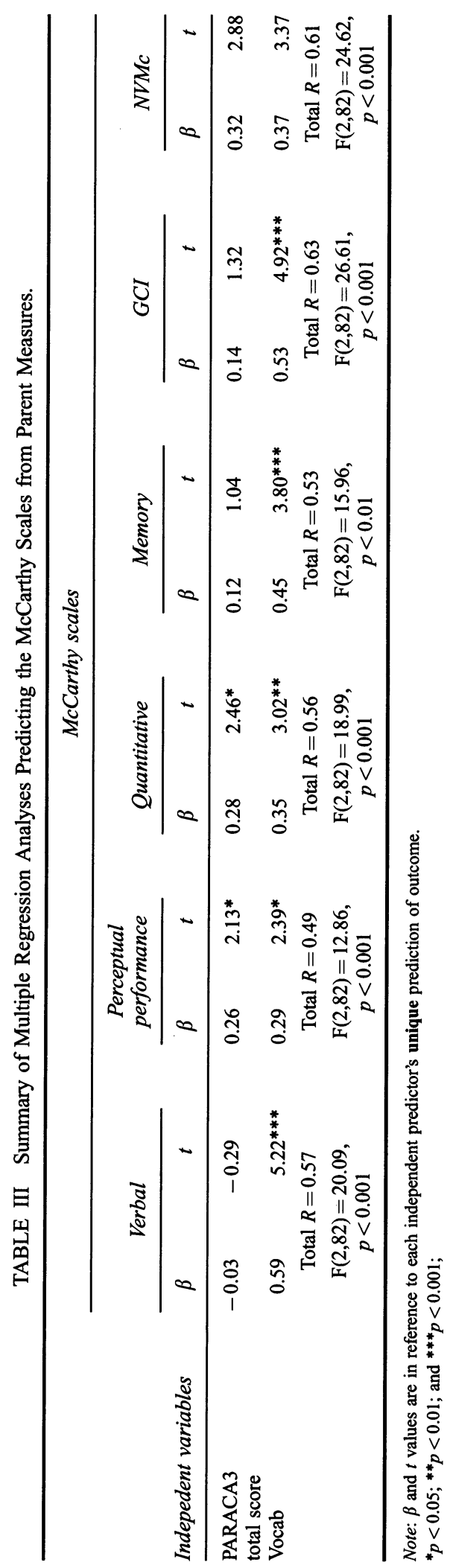


The correlation and regression analyses indicated the criterion validity of the PARCA3 and vocabulary. The correlations showed that both components of the PARCA3 (parent-report and parent-administered) were substantially associated with the McCarthy scales, as was the vocabulary measure. The non-verbal PARCA 3 measure was most highly correlated with the non-verbal McCarthy scales, as expected. The regression analyses showed that the verbal and non-verbal parent-based measures each uniquely predict most of the McCarthy scales. Most importantly, the two measures in combination significantly and substantially predict the GCI (Total $R=0.63$ ). This is in keeping with other validation studies using tester-administered assessments. For example, Harrison and Wiebe (1977) report correlations of 0.45 and 0.56 between the McCarthy and Stanford-Binet for a sample of 59 children between $2 \frac{1}{2}$ and 6 years old, and Krohn and Traxler (1979) found correlations between 0.48 and 0.82 between the McCarthy and four other tester-administered measures of general cognitive ability, for 46 children aged between $2 \frac{1}{2}$ and 5 years old.

The examination of the discriminant validity of the measures produced somewhat unexpected results: the non-specificity of the vocabulary measure went against our specificity hypothesis. Vocabulary was more highly associated with all the McCarthy scales than was PARCA3. However, as foreshadowed by the correlation results, the vocabulary measure not only significantly predicted all of the McCarthy scales, but further, the prediction was higher than PARCA3 in every case. It seems that the influence of vocabulary simply overwhelms the effect of non-verbal abilities. Although these results were not what we anticipated, in fact, evidence from the standardization sample of the McCarthy at the same age mirrors our findings. The verbal scale is reported to correlate 0.94 with the GCI, clearly showing just how good an indicator of general intelligence verbal abilities are during the preschool years. It is important to emphasize, however, that as expected, the PARCA3 provided unique prediction for non-verbal McCarthy scales, including the non-verbal composite. This underscores the added value of the PARCA3 in addition to a measure of vocabulary at this age.

The question remains why the pattern of results was different at age two from those at age three. At age two, although vocabulary correlated highly with general cognitive ability as assessed by the Bayley MDI, the PARCA provided unique prediction of $g$ (Saudino et al., 1998). At age three this independent prediction by the PARCA3 was lost. Language research has demonstrated that the developmental period between two and three years of age is a period of change in language production (Barrett, 1999). For example there is an explosion of vocabulary words, and a particular characteristic developed during this period is the "fast mapping" of words onto meanings (Nelson, 1988). Thus, it could be argued that verbal abilities are qualitatively different at 3 and 4 years from those at 2 years. It could be that the vocabulary of the older child is more highly associated with $g$ than non-verbal ability due to the fact that vocabulary incorporates quite complex cognitive processes that are especially good indices of $g$.

Emerging genetic findings from the full 1994 cohort of TEDS are consistent with the developmental emergence of heritable $g$ (Price et al., in prep.). Genetic influences from verbal ability at two years are more strongly related to genetic influences on non-verbal ability at three years than the converse. This suggests that verbal ability in infancy may be more predictive genetically of later $g$ than is non-verbal ability, thus informing the present findings.

Together with evidence for the validity of the original two-year-old PARCA and the MacArthur Communicative Development Inventories: UKSF adaptation (Saudino et al., 1998), the present study confirms the usefulness of parent-based measures of young children's abilities. Despite the limited power given the relatively small sample size, these findings are convincing in terms of the large effect sizes as well as statistical significance. The results are also bolstered by the population-based sample in which participants are 
representative of parents in England and Wales. In addition, the ongoing use of PARCA3 within the TEDS study (about 10,000 twin pairs) indicates that parents across the spectrum of the population can use PARCA3 to assess their children's abilities. Indeed, previous evidence on the validity of parent report and its relationship with parental education (Dale, 1996) suggests that parent reports are valid over a wide, but not universal, range of education levels.

In conclusion, parents not only have a unique opportunity to observe and assess their children's performance in their natural environment over much time and many situations, but their assessments are valid. The usefulness of parental reports is questioned due to fears of bias, inaccuracy, and a lack of frame of reference from which to assess the child's developmental level. PARCA3 with a vocabulary measure provides an inexpensive yet valid and reliable measure of cognitive ability for young children in a research context. PARCA and PARCA3 and vocabulary measures suggest that individual testing sessions with trained researchers may be unnecessary, thus potentially freeing up valuable time and scarce funding resources. Rather than thinking about parent-based measures as poor cousins of tester-based measures, we suggest that these parent-based measures might be more indicative of ability, a hypothesis that can be tested as we continue to follow children in the full TEDS sample into the early school years.

A caveat to these conclusions is, however, required. Parental assessments, although valid, may nevertheless be prone to bias. For example, parental intelligence or education may influence the assessments. The modest amount of evidence available on parental education and validity of parent report (Diamond and Squires, 1993; Dale, 1996) suggests that parent report is valid over a wide social-class range, though it may be reduced for parents with less than a high school education. Similarly, the accuracy of parental reports might be distorted in certain clinical contexts when parents may have some desire to prove that their child does not have a problem or in certain educational contexts in which parents might be motivated to exaggerate their children's abilities. Finally, there is a lack of information regarding the role of potential cultural differences in affecting parent report. There are welldocumented differences in views of children and child rearing, views of disabilities and their causes, and views of medicine and healing that might affect either parental observation or reporting of their children's abilities (Hanson, Lynch and Wayman, 1990). We would caution that the use of parent report measures, such as the PARCA3, as clinical instruments are inappropriate until the issues of potential parental bias are adequately addressed. Clearly, we were not able to explore these issues in our nonclinical sample, but the demonstrated validity of the PARCA3 in the present study suggests that parent reports of nonverbal cognitive ability will be useful as a research tool for screening cognitive development in young children.

\section{References}

Barrett, M. (Ed.) (1999) The Development of Language. East Sussex, UK: Psychology Press.

Bayley, N. (1993) Bayley Scales of Infant Development (2nd ed.). San Antonio, TX: Psychological Corporation.

Bornstein, M. H. and Haynes, O. M. (1998) Vocabulary competence in early childhood: measurement, latent construct, and predictive validity, Child Development, 69, 654-671.

Bricker, D. and Squires, J. (1989) The effectiveness of parental screening of at-risk infants: The Infant Monitoring Questionnaires, Topics in Early Childhood Special Education, 9, 67-85.

Bricker, D., Squires, J. and Mounts, L. (1995) Ages and Stages Questionnaires: A Parent-Completed ChildMonitoring System. Baltimore, MD: Brooks.

Dale, P. S. (1996) Parent report assessment of language and communication. In: Cole, K. N., Dale, P. S. and Thal, D. J. (Eds.), Assessment of Communication and Language. Baltimore: Paul Brookes, pp. 161-182.

Dale, P. S., Reznick, J. S. and Thal, D. J. (1998) A parent report measure of language development for threeyear-olds. International Conference on Infant Studies, Atlanta, Georgia. 
Dale, P. S., Simonoff, E., Bishop, D. V. M., Eley, T. C., Oliver, B., Price, T. S., Purcell, S., Stevenson, J. and Plomin, R. (1998) Genetic influence on language delay in two-year-old children, Nature Neuroscience, 1, 324-328.

Diamond, K. E., and Squires, J. (1993) The role of parental report in the screening and assessment of young children, Journal of Early Intervention, 17, 107-115.

Fenson, L., Dale, P. S., Reznick, S. J., Bates, E., Thal, D. J. and Pethick, S. J. (1994) Variability in early communicative development, Monographs of the Society for Research in Child Development, 59(242).

Fenson, L., Pethick, S. and Cox, J. L. (1994) The MacArthur Communicative Development Inventories: Short Form Versions. Unpublished manuscript, San Diego State University.

Hanson, M. J., Lynch, E. W. and Wayman, K. I. (1990) Honoring the cultural diversity of families when gathering data, Topics in Early Childhood Education, 10, 112-131.

Harrison, K. A. and Wiebe, M. J. (1977) Correlational study of McCarthy, WISC, and Stanford-Binet scales, Perceptual and Motor Skills, 44, 63-68.

Ireton, H. and Thwing, E. (1974) The Minnesota Child Development Inventory. Minneapolis, MN: Behavior Science Systems.

Krohn, E. J. and Traxler, A. J. (1979) Relationship of the McCarthy Scales of Children's abilities to other measures of preschool cognitive, motor and perceptual development, Perceptual and Motor Skills, 49, 783-790.

McCarthy, D. (1972) McCarthy Scales of Children's Abilities. New York: The Psychological Corporation.

Nelson, K. (1988) Constraints on word learning? Cognitive Development, 3, 221-246.

Price, T. S., Dale, P. S., Stevenson, J., Eley, T. C. and Plomin, R. (in prep.) The Development of General Cognitive Ability Inpre-School Age Children.

Sattler, J. M. (1988) Assessment of Children (3rd ed.). San Diego, CA: J.M. Sattler. Social, Genetic and Developmental Psychiatry Research Centre.

Saudino, K. J., Dale, P. S., Oliver, B., Petrill, S. A., Richardson, V., Rutter, M., Simonoff, E., Stevenson, J. and Plomin, R. (1998) The validity of parent-based assessment of the cognitive abilities of two-year-olds, British Journal of Developmental Psychology, 16, 349-363.

Thorndike, R. L., Hagen, E. P. and Sattler, J. M. (1986) The Stanford-Binet Intelligence Scale. Chicago, IL: The Riverside Publishing Company.

Wechsler, D. (1989) Wechsler Preschool and Primary Scales of Intelligence-Revised. San Antonio, TX: Psychological Corporation.

\section{APPENDIX: RESULTS FOR FIRST- AND SECOND- BORN TWINS SEPARATELY}

TABLE A Correlations Between Parent Measures and the McCarthy Scales for First-Born and (SecondBorn) Twins.

\begin{tabular}{lcccc}
\hline \multicolumn{5}{c}{ PARCA3 } \\
\cline { 2 - 5 } & Parent-report & Parent-administered & PARCA3 total score & Vocab \\
\hline Verbal & $0.33^{*}$ & $0.27^{\mathrm{T}}$ & $0.36^{*}$ & $0.68^{* * *}$ \\
& $\left(0.30^{\mathrm{T}}\right)$ & $(0.16)$ & $\left(0.30^{\mathrm{T}}\right)$ & $\left(0.48^{* *}\right)$ \\
Perceptual Performance & $0.36^{*}$ & $0.38^{*}$ & $0.45^{* *}$ & $0.48^{* *}$ \\
& $\left(0.45^{* *}\right)$ & $(0.17)$ & $\left(0.40^{* *}\right)$ & $\left(0.40^{* *}\right)$ \\
Quantitative & $0.50^{* *}$ & $0.43^{* *}$ & $0.56^{* * *}$ & $0.66^{* * *}$ \\
& $\left(36^{*}\right)$ & $\left(0.29^{\mathrm{T}}\right)$ & $\left(0.42^{* *}\right)$ & $\left(0.37^{*}\right)$ \\
Memory & $0.53^{* * *}$ & $0.39^{* *}$ & $0.56^{* * *}$ & $0.72^{* * *}$ \\
& $(0.20)$ & $(0.17)$ & $(0.24)$ & $\left(0.35^{*}\right)$ \\
NVMc & $0.48^{* *}$ & $0.46^{* *}$ & $0.56^{* * *}$ & $0.63^{* * *}$ \\
& $\left(0.51^{* *}\right)$ & $\left(0.28^{*}\right)$ & $\left(0.50^{* *}\right)$ & $\left(0.47^{* *}\right)$ \\
GCI & $0.43^{* *}$ & $0.38^{*}$ & $0.49^{* *}$ & $0.70^{* * *}$ \\
& $\left(0.44^{* *}\right)$ & $(0.24)$ & $\left(0.44^{*}\right)$ & $\left(0.53^{* * *}\right)$ \\
\hline
\end{tabular}

Note: First-born $(N=43)$ coefficients are above the (second-born) $(N=42)$ coefficients. ${ }^{\mathrm{T}} p<0.10 ;{ }^{*} p<0.05 ;{ }^{* *} p<0.01 ;{ }^{* * *} p<0.001$. 


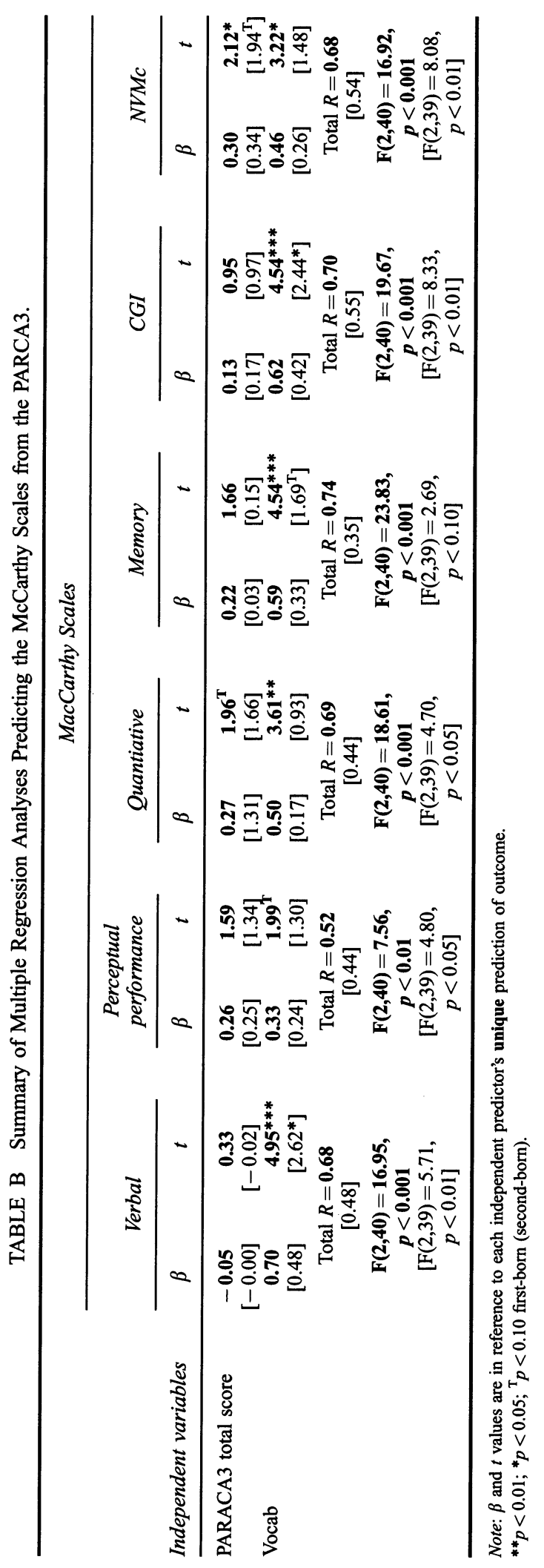

